\title{
Absence of a redundant-signals effect in a reaction time task with divided attention
}

\author{
G. ROBERT GRICE, LYN CANHAM, and JOHN W. GWYNNE \\ University of New Mexico, Albuquerque, New Mexico
}

\begin{abstract}
In letter-identification reaction time experiments, a redundant-signals advantage was obtained when one location contained an irrelevant letter on single-target trials. It was not obtained when the position was blank. This confirms a prediction from a previous analysis in terms of variable criterion theory. The intercept of the linear function relating the growth of excitatory strength for redundant targets to that for the sum of single-target strengths is determined by the presence or absence of an irrelevant distractor. This confirms a second prediction. In this task, the redundantsignals advantage is due to distraction from an irrelevant stimulus occupying a relevant location. There is also evidence for a smaller distraction effect even from a redundant target. These findings are inconsistent with predictions of separate activation theory. Apparent differences between these results and those of tachistoscopic recognition experiments are discussed.
\end{abstract}

The redundant-signals, or redundant-targets, effect refers to the advantage in performance that is obtained with multiple redundant-stimulus presentation when compared with the presentation of a single stimulus. This effect has been investigated and observed in a variety of experimental situations. The specific concern here is with reaction time ( $R T$ ) experiments of letter identification, in which the target letter may occur in either, or both, of two locations in a visual display. Such experiments have recently been used by Miller (1982) and by Grice, Canham, and Boroughs (1984) in investigations of the theoretical basis of the redundant-signals effect.

Miller (1982) distinguishes between two classes of relevant theories-separate activation and coactivation. According to separate-activation theories, redundant inputs are processed in parallel and the response occurs when the target is detected in one channel. If the two processes overlap, statistical facilitation will result (Raab, 1962). According to the coactivation view, redundant inputs contribute to the growth of a single state of strength of activation. Multiple inputs produce faster growth than do single stimuli, resulting in quicker response. Miller also devised a test capable of evaluating separate activation models. His experiments were yes-no RT experiments, with the target letter presented in either of two locations, in both locations, or neither. The test resulted in the rejection of the class of separate activation models for conditions in which the location without a target was filled with an irrelevant noise letter.

The experiments of Grice et al. (1984) were choice reaction time (CRT) experiments with two target letters, each mapped on a different response. The purpose of this was to eliminate a possible deficiency of the yes-no proce-

Requests for reprints should be addressed to G. Robert Grice, Department of Psychology, University of New Mexico, Albuquerque, NM 87131. dure. They reported five such experiments. The results, in general, confirmed Miller's conclusion concerning the inadequacy of separate-activation theory. In addition, these authors investigated the principle by which the strengths from separate stimuli combine in the case of redundant presentation. Applying the scaling procedures of variable criterion theory to the data of the cumulative RT distribution functions, they determined functions for the growth of excitatory strength during the time following stimulus onset. The results were surprisingly precise and consistent. In each experiment, throughout the entire distribution, excitatory strength for redundant presentation grew as a precise linear function of the sum of the strengths produced by the two separate stimuli at the same time. The slopes ranged from .489 to .524 . All of the linear functions contained a positive, additive constant. It was pointed out that the additive constant was not an artifact of an arbitrary origin of the strength scale. Since the summation relationship was actually approximately an average, the constant would be present for any reasonable origin. The redundant-signals effect was dependent on a constant increment throughout the distribution and not the summation principle.

It was suggested that the presence of an irrelevant noise letter in the position with no target on single-target trials produced a distraction decrement, constant through the distribution. This agrees with the finding of Grice, Canham, and Schafer (1982), who found that an irrelevant noise letter in a focused-attention task also produced a constant decrement. On two target trials, the distractor is absent, so what is being summed, or averaged, is the single target strengths plus the amount of the distraction decrement. This accounts for the additive constant. Now, if the "redundant-signals effect" is dependent on the presence of an irrelevant letter, the clear prediction follows that the effect would not be obtained when the redundant signals condition was compared with trials consist- 
ing of a single target alone. This prediction is somewhat counterintuitive, first, because of the way this situation has been conceptualized in the past. Second, there is clear evidence that the effect can be obtained, in terms of percent correct, in detection experiments with brief exposure and no noise letters present (Eriksen, 1966; Eriksen \& Lappin, 1965). The experiments here are tests of this prediction.

\section{EXPERIMENT 1}

\section{Method}

Procedure. The first experiment was, in part, a replication of Experiment 3 reported by Grice et al. (1984), but it was expanded to include presentations of single targets alone. Except for changes necessary to include these trials, the details of the procedures were the same as those described there.

The letter stimuli were the normal, double-width characters of the TRS-80 Model I microcomputer. When two letters were presented, they were separated horizontally by $6^{\circ}$. When a target was presented alone, it occupied one of the same positions. A plus sign, used as a fixation point, was located midway between the two letter positions and was on the screen continuously except when letters were present. The target letters were $H$ and $S$, each occurring equally often, and the irrelevant letter, when present, was $Y$. There were five kinds of trials, presented equally often. The trial types were as follows: target in both positions, target right and irrelevant letter left; target left and irrelevant letter right; target alone right; and target alone left.

Responses were made with two telegraph keys operated with the right and left forefingers. One half of the subjects were instructed to respond to $\mathrm{H}$ with the right key and $\mathrm{S}$ with the left key; the other half had the reverse pairing. A .5-sec auditory warning signal preceded each trial by $1 \mathrm{sec}$. The display went off when the subject responded. The instructions were to respond to the target as quickly as possible while avoiding errors. Visual and auditory feedback was given for errors. RTs were recorded in milliseconds. The interval between trials was $4 \mathrm{sec}$. The order of trial types and targets was irregular. Since the stimulus-response compatibility effect is strong in this situation, single-target trials are not analyzed in terms of the right and left positions, but in terms of whether the target and response were, or were not, on the same side.

The experiment began with 50 practice trials followed by a short rest, during which additional instruction was given if necessary. There then were 250 trials without interruption. Data are based on the last 240 trials, 48 with each kind of trial.

Subjects. The subjects were 28 undergraduate women from courses in introductory psychology. They received course credit for participation.

\section{Results}

Mean reaction time. The data are first presented in terms of means and standard deviations of subject mean RTs for the five experimental conditions. These data are presented in Table 1. Error rates were low. For the onetarget-alone conditions, the rates were .012 for compatible and .031 for incompatible. For the distractor conditions, they were .010 for compatible and .035 for incompatible. The error rate for the two-target condition was .016 .

In an analysis of the single-target conditions, it is clear that the irrelevant noise letter did produce a distraction effect, as indicated by slower mean RTs. The effect was significant $[\mathrm{F}(1,27)=95.63, \mathrm{p}<.001]$. As expected,
Table 1

Means and Standard Deviations of Correct Mean Reaction Time in Milliseconds

\begin{tabular}{|c|c|c|c|c|c|}
\hline \multirow[b]{2}{*}{ Measure } & \multicolumn{2}{|c|}{ No Distractor } & \multicolumn{2}{|c|}{ With Distractor } & \multirow[b]{2}{*}{$\begin{array}{c}\text { Both } \\
\text { Positions }\end{array}$} \\
\hline & $\begin{array}{c}\text { Compat- } \\
\text { ible }\end{array}$ & $\begin{array}{l}\text { Incompat- } \\
\text { ible }\end{array}$ & $\begin{array}{c}\text { Compat- } \\
\text { ible }\end{array}$ & $\begin{array}{l}\text { Incompat- } \\
\text { ible }\end{array}$ & \\
\hline Mean & 431 & 469 & 470 & 507 & 447 \\
\hline SD & 66 & 65 & 65 & 73 & 57 \\
\hline
\end{tabular}

the S-R compatibility effect was substantial and significant $[\mathrm{F}(1,27)=92.49, \mathrm{p}<.001]$. There was almost no interaction between these two effects $[F(1,27)<1]$. In other words, the size of the compatibility effect and the size of the distraction effect were each unaffected by the other variable.

In evaluating the redundant-targets effect, the proper comparison is between the conditions with targets in both postions and the faster of the two single-target conditions, that is, with the target in the compatible position. When this comparison is made with the distractor condition, the two-target condition is significantly faster $[\mathrm{t}(27)=3.794$, $\mathrm{p}<.001]$. This replicates the redundant-signals effect obtained in the previous experiments with an irrelevant letter present. However, the redundant signals effect is not observed when the comparison is made with the nodistractor condition. In this comparison, the compatible single-target condition is actually faster than that with two targets. This difference is significant $[\mathrm{t}(27)=3.035$, $\mathrm{p}<.01]$. Thus, the experiment not only confirms our prediction, but provides additonal information. On two target trials, there is always a target in the compatible location. However, these results indicate that even a redundant target in the incompatible position produces a small, but significant, distraction effect. Of course, this effect is considerably smaller than that produced by an irrelevant letter.

Scaling analysis. A scaling analysis of the data of the cumulative RT distributions has been conducted in the same manner as those that led to the present prediction (Grice et al., 1984). This is based on the conception of variable criterion theory, that the excitatory strength produced by a stimulus grows as an orderly function of time following onset until criterion strength is reached. The assumption of a normally distributed criterion leads to the measurement of these functions in a Thurstonean metric. The distributions analyzed were average cumulative distributions obtained by the method of Ratcliff (1979). For each subject separately, quantiles were obtained at .05 intervals. These quantiles were then averaged over subjects. As in the previous analyses, the subject distributions were first corrected for errors by applying the equations of the race model of Grice, Spiker, and Nullmeyer (1979).

The scaling analysis resulted in smooth, negatively accelerated functions for the growth of excitatory strength (E) for each of the five conditions. The functions for the four single target conditions were fitted with exponential growth functions of the same kind as in the previous ex- 
periments (Grice et al., 1984). The fits were excellent. In all cases they resulted in fits to the obtained distributions in which the proportion of variance accounted for was .999 or greater. Graphs of the fitted functions are presented in Figure 1. The unit normal functions on the strength axis represent the criterion distribution and illustrate the scaling model. The cumulative proportion of responses at any time is given by the area of the normal curve lying below the ordinate of a growth function at that time.

The relation between the redundant-targets condition and the sum of the strengths of the single-target conditions was examined in the same manner as previously. The strengths of the single-target conditions were obtained by calculating the values of the fitted functions at the times indicated by the 19.05 quantiles of the two-target condition. The obtained scale values for the two-target conditon were then plotted against the sum of the two singletarget conditions. The relation was obtained separately for the distractor and no-distractor conditions. The data are plotted in Figure 2. In each instance, the result was a precise linear relation. The linear regression equation for the distractor conditions was as folows:

$$
\mathrm{E}_{\text {BOTH }}=.513\left(\mathrm{E}_{\text {COMPATIBLE }}+\mathrm{E}_{\text {INCOMPATIBLE }}\right)+.389 .
$$

The value of $r^{2}$ was .999 . This replicates the results of the previous experiments with irrelevant noise letters, including a slope near .5 and a positive intercept. The regression equation for the conditions with no distractor was as follows:

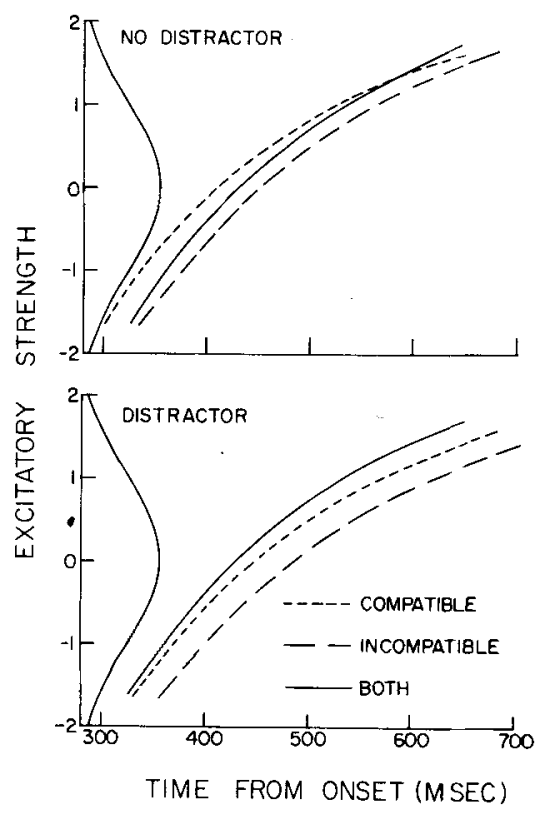

Figure 1. Calculated functions for the growth of excitatory strength for each experimental condition. The function for targets in both positions is included in both the upper and lower panels. Normal functions on the strength axis represent the criterion distribution.

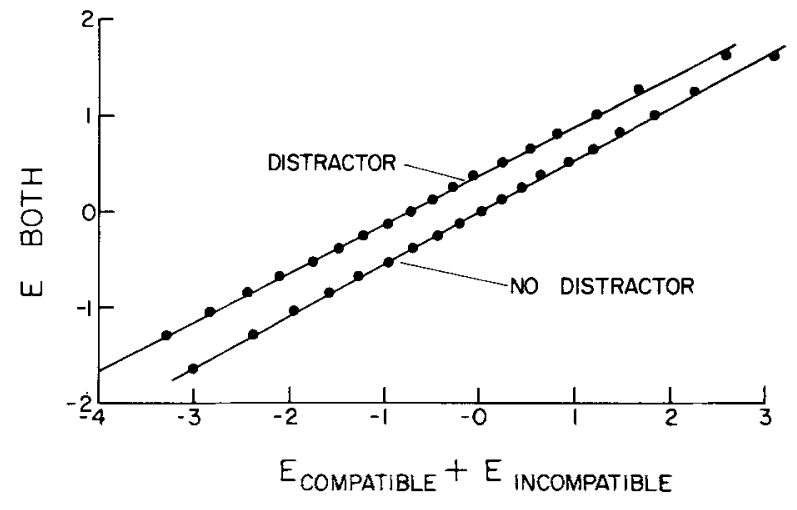

Figure 2. Relations between the strength of the condition with targets in both locations and the sum of the strengths of the single-target conditions with and without the distractor. Points are at the 19.05 quantiles of the redundant-target condition. Lines are least squares regression lines.

$$
E_{\text {BOTH }}=.547\left(E_{\text {COMPATIBLE }}+E_{\text {INCOMPATIBLE }}\right)+.011
$$

Again, $\mathrm{r}^{2}$ was .999 . The basis for the present prediction was the hypothesis that the intercepts of such linear equations were dependent on distraction or interference produced by the irrelevant noise letter on single-target trials. The results clearly confirm that hypothesis. The small difference in the slopes reflects the fact the $E$ was greater at short latencies in the no-distractor conditions, but that the functions were approaching comparable limits.

The growth function for the condition with the target in both positions may be computed with equal accuracy from either Equation 1 or Equation 2. The function is presented in Figure 1 for comparison with the singletarget conditions. In the upper panel, it is computed from Equation 2; in the lower panel, it is computed from Equation 1 . For the distractor condition, it is consistently above the response-compatible condition. In the no-distractor condition, the compatible function is superior until about 560 msec.

The five cumulative distribution functions on which the analysis was based are presented in Figure 3. According to the scaling model, the smooth functions were calculated by obtaining the cumulative normal function of the equations fitted to the single-target conditions at the 19 quantiles. For the condition with both targets, the function used was that obtained from Equation 1 or Equation 2. In all instances, the proportion of variance accounted for was .999 or greater.

\section{EXPERIMENT 2}

A second expeiment has been conducted in order to evaluate some possible interpretations of the first experiment. For example, it is possible that the above results are specific to situations involving the $S-R$ compatibility variable. In the redundant-targets condition, one target might become clear sooner than the other. This could be produced by eye fixation, attention bias, or just random 


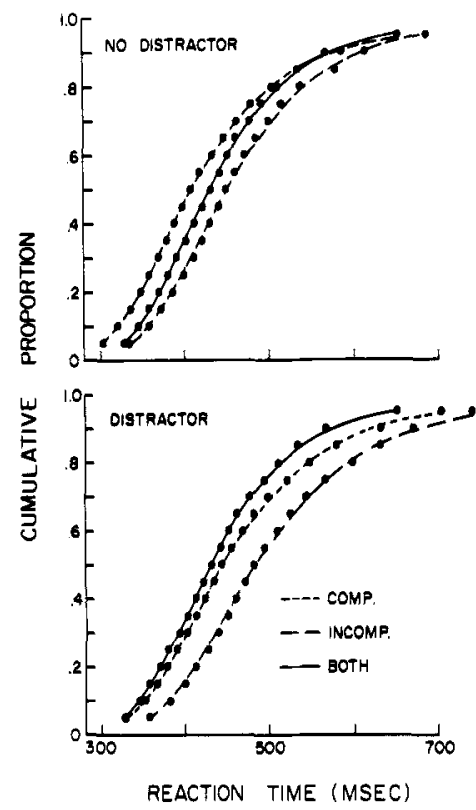

Figure 3. Calculated and obtained cumulative distributions for a single target in compatible and incompatible positions. The distribution for target in both positions is presented in each panel.

variability in the visual system. If this were true, responses on redundant-target trials might be a mixture of compatible and incompatible responses, thus attenuating the redundant-targets effect when compared with compatible trials with no distractor. To eliminate this possibility, we conducted an experiment with vertically spaced stimuli-a condition that has been shown not to produce a compatibility effect (Wallace, 1971; Grice et al., 1984). To eliminate the possibility of slow responses to stimuli seen following a saccade, we also shortened the stimulus exposure to make this impossible. The experiment was a replication of Experiment 5 by Grice et al. (1984) with the addition of the no-distractor condition and a shortened stimulus exposure.

\section{Method}

The procedures were the same as in the preceding experiment with two exceptions. The two letters were presented equidistant from the fixation point directly above and below it. The vertical distance between the two letters was approximately $3^{\circ}$. The stimulus exposure duration was $150 \mathrm{msec}$. There were 28 female subjects from the same populations as above.

\section{Results}

Means and standard deviations of subject mean RTs for the five experimental conditions are presented in Table 2. Error rates were again low. For the upper position, the mean rates were .016 without a distractor and .023 with a distractor present. For the lower position, the corresponding rates were .018 and .011 . The rate for the redundant-targets condition was .013 .

As in the previous experiment, the irrelevant noise letter produced a significant distraction effect. In an analy- sis of the single-target RTs, for the distractor effect, $F(1,27)=33.47, p<.001$. The effect of target location was not significant $[F(1,27)=1.07$, n.s. $]$. Also, the interaction of distraction and location was not significant $[\mathrm{F}(1,27)<1]$. The distraction effect was essentially unaffected by the change to vertical spacing and the shortened exposure. The average effect was $38 \mathrm{msec}$ in the first experiment and $34 \mathrm{msec}$ in the second.

In comparing the redundant-targets condition with the single-target distractor conditions, it is significantly faster than the faster, upper position $[\mathrm{t}(2,7)=4.967$, $p<.001]$. This confirms both the finding of the first experiment and that of Grice et al. (1984) with the same spatial arrangement. The redundant-targets condition is not faster than either target position with no distractor. Thus, the major findings of the first experiment are confirmed.

The additional evidence for a small distraction effect of a redundant target requires a different analysis. Since some subjects are faster to one target and some are faster to the other, the consistency of this effect must be evaluated by comparing the redundant-targets condition with the faster of the no-distractor positions for each subject separately. This was not necessary with the compatibility effect present. The mean RT for the faster, nodistractor position of each subject was $427 \mathrm{msec}, 10 \mathrm{msec}$ faster than that for the redundant-targets condition. This difference is significant $[\mathrm{t}(27)=3.883, \mathrm{p}<.001]$. This confirms the finding of a small, but significant, distraction effect of a redundant target in the subject's less preferred position. The mean of the faster distractor position was $456 \mathrm{msec}$, significantly slower than the redundant-targets condition $[\mathrm{t}(27)=3.058, \mathrm{p}<.01]$.

A similar analysis based on medians, or other quantiles, is also of interest because it tests a clear prediction of separate activation theories involving a principle of statistical facilitation. Miller (1982) showed that such theories require that at no point in the cumulative distribution functions may the probability of response to redundant targets exceed the sum of the probabilities of response to the separate channels. Relevant to the present data, there is another prediction that follows with equal rigor. At no point in the cumulative distribution functions may the probability of response to either of the separate channels be greater than the probability of response to redundant targets. This means that no quantile point in the faster channel may be reached before that quantile is reached with redundant targets. As in the case of Miller's test, this principle may be examined for each subject, and

Table 2

Means and Standard Deviations of Correct Mean Reaction Time in Milliseconds for the Upper and Lower Target Positions

\begin{tabular}{|c|c|c|c|c|c|}
\hline \multirow[b]{2}{*}{ Measure } & \multicolumn{2}{|c|}{ No Distractor } & \multicolumn{2}{|c|}{ With Distractor } & \multirow{2}{*}{$\begin{array}{c}\text { Both } \\
\text { Positions }\end{array}$} \\
\hline & Upper & Lower & Upper & Lower & \\
\hline $\mathrm{Me}$ & 433 & 437 & 468 & 471 & 437 \\
\hline SD & 68 & 69 & 81 & 82 & 69 \\
\hline
\end{tabular}


the significance of any violation evaluated by a t test. Actually, the principle is violated throughout the distributions until the .9 quantiles, but we have tested for signficance only at the medians. The mean of the subject medians for the faster position with no distractor is $413 \mathrm{msec}$; the mean of the medians for redundant targets is $426 \mathrm{msec}$. This difference is significant $[\mathrm{t}(27)=4.236$, $\mathrm{p}<.001]$. As would be expected, a significant violation also occurred in the first experiment with horizontally spaced stimuli. The mean of the median RTs for the faster position with no distractor was $409 \mathrm{msec}$ and that for the redundant targets condition was $433 \mathrm{msec}[\mathrm{t}(27)=4.877$, $\mathrm{p}<.001]$. Thus, both experiments require that separate activation theory must be rejected. The median data also confirm the distraction effect produced by the presence of a redundant target.

Growth functions for the single-target conditions in the second experiment were based on the faster position for each subject in each of the distractor conditions. The resulting four functions were fitted with exponential growth functions with precision equal to that of the first experiment. The redundant-target function was again linearly related to the sum of the single-target functions for both the no-distractor and distractor conditions. The values of $r^{2}$ were .997 for the no-distractor condition and .998 for the irrelevant-letter condition. For the nodistractor condition, the slope of the least squares regression line was .529 and the intercept was -.046 . For the distractor condition, the slope was .556 and the intercept was .336. These data again confirm the prediction that the intercept of the linear relation is determined by the presence or absence of an irrelevant distractor. The use of vertically spaced stimuli and the brief stimulus exposure produced results in full agreement with those of the first experiment.

\section{DISCUSSION}

Using the information in RT distributions, Grice et al. (1984) sought to determine the principle by means of which redundant stimuli combine in strength in RT tasks with divided attention. They did discover the principle, which was remarkably precise, consistent throughout the distribution, and quite general, in that it applied to a variety of visual displays. However, the relation turned out to be more nearly an averaging principle than a summation principle sufficient to account for the advantage achieved with redundant targets. That advantage was dependent on a constant through the distribution. Partly on the basis of similar findings by Grice et al. (1982), they hypothesized that this was the result of distraction produced by an irrelevant noise letter occupying one of the relevant positions in single-target conditions. This led to the prediction that the constant, the intercept of the linear function, would be eliminated or substantially reduced when single targets were presented without noise letters present. This further implied that the redundant- target advantage would not be present in such a comparison. That both of these predictions were confirmed increases confidence in the utility and validity of the analysis based on variable criterion theory.

The distraction decrement produced by even a redundant target is a new finding that may be a bit surprising. However, we have recently obtained, in unpublished research, a similar result with a focused-attention task. When the target was flanked by identical noise letters, RT was slower than in a no-noise condition. This decrement was also less than that produced by an irrelevantnoise letter.

These experiments point to an area in which, apparently, different principles apply to recognition experiments with brief, tachistoscopic exposure and RT recognition experiments. Eriksen and Lappin (1965), using brief exposures, found that percent correct recognition increased monotonically as the number of target letters was increased from one to six. Eriksen (1966) presented either one or two target letters in one or two of four uncertain locations, and found increased recognition with redundant targets. In neither of these experiments were noise letters ever present in the display. In both experiments, the authors found the data to be consistent with the interpretation that the advantage depended on multiple, independent opportunities to detect the target. The situation is different with CRT. When the redundant-signals effect was obtained in a comparison with noise-letter conditions, Grice et al. (1984) found that an independence interpretation had to be rejected for all five of their experiments. Now, we find no such advantage relative to a no-noise condition. ${ }^{1}$

It is important to observe instances in which these two different classes of experiments lead to different conclusions. However, such instances should not be the source of great surprise. In RT experiments, the subject responds under speed pressure with the stimulus still present or following a sufficiently large exposure to produce high accuracy. According to both variable criterion theory and the continuous flow model of Eriksen and Shultz (1979), the associative strength or response priming produced by the stimulus continues to grow until the criterion is reached. In the typical tachistoscopic experiment, the observer does not respond until whatever perceptual growth possible is complete. Even when RT measures are taken in such experiments, RTs are considerably longer than in conventional CRT experiements with accurate performance. In discussing the difference between these tasks, Smith (1968) suggested that both principles of recognition and those of judgment may differ in the two situations. More recently, Santee and Egeth (1982) have obtained differing relations between comparable experimental conditions for percent correct measures with brief exposures and RT measures with long exposures.

The present series of experiments clearly favor a form of coactivation theory. With redundant signals present, each target contributes about half of its strength con- 
sistenly through the entire period of the RT distributions. This has been the consistent finding in seven experiments. However, this form of coactivation does not produce a redundant-signals advantage.

\section{REFERENCES}

ERIKSEN, C. W. (1966). Independence of successive inputs and uncorrelated error in visual form perception. Journal of Experimental Psychology, 72, 26-35.

ERIKSEN, C. W., \& LAPPIN, J. S. (1965). Internal perceptual system noise and redundancy in simultaneous inputs in form identification. Psychonomic Science, 2, 351-352.

ERIKSEN, C. E., \& ShULTZ, D. W. (1979). Information processing in visual search: A continuous flow conception and experimental results. Perception \& Psychophysics, 25, 249-263.

Grice, G. R., Canham, L., \& Boroughs, J. M. (1984). Combination rule for redundant information in reaction time tasks with divided attention. Perception \& Psychophysics, 35, 451-463.

Grice, G. R., Canham, L., \& Schafer, C. (1982). Development of associative and perceptual interference. Perception \& Psychophysics, 32, 375-387.

Grice, G. R., SPIKeR, V. A., \& Nullmeyer, R. (1979). Variable criterion analysis of individual differences and stimulus similarity in choice reaction time. Perception \& Psychophysics, 25, 353-370.

Miller, J. (1982). Divided attention: Evidence for coactivation with redundant signals. Cognitive Psychology, 14, 247-279.
RAAB, D. (1962). Statistical facilitation of simple reaction times. Transactions of the New York Academy of Sciences, 24, 574-590.

RATCLIFF, R. (1979). Group reaction time distributions and an analysis of distribution statistics. Psychological Bulletin, 86, 446-461.

SANTEE, J. L., \& EGETH, H. E. (1982). Do reaction time and accuracy measure the same aspects of letter recognition? Journal of Experimental Psychology: Human Perception and Performance, 8, 489-501.

Smith, E. E. (1968). Choice reaction time: An analysis of the major theoretical positions. Psychological Bulletin, 69, 77-110.

WALLACE, R. J. (1971). S-R compatibility and the idea of a response code. Journal of Experimental Psychology, 88, 354-360.

\section{NOTE}

1. In one of his yes-no experiments, Miller (1982) included a nonoise condition. In that case, the redundant-targets condition was a little faster than the mean of the faster of the single-target positions. However, in this situation, some subjects will be faster to one position and some to the other. In this instance, Miller did not present data in which the redundant-targets condition was compared with the faster position for each subject separately. It appears unlikely that this procedure would have shown a redundant-targets advantage. Miller made no claim that such an advantage had been demonstrated.

(Manuscript received August 3, 1984; revision accepted for publication November 12, 1984.) 